\title{
Geometric Properties of Cesàro Averaging Operators
}

\author{
Priyanka Sangal and A. Swaminathan \\ Department of Mathematics, Indian Institute of Technology (IIT) Roorkee, Uttarakhand 247667, India \\ Correspondence should be addressed to Priyanka Sangal; sangal.priyanka@gmail.com
}

Received 10 August 2017; Accepted 9 November 2017; Published 28 November 2017

Academic Editor: Pranay Goswami

Copyright (C) 2017 Priyanka Sangal and A. Swaminathan. This is an open access article distributed under the Creative Commons Attribution License, which permits unrestricted use, distribution, and reproduction in any medium, provided the original work is properly cited.

\begin{abstract}
Using positivity of trigonometric cosine and sine sums whose coefficients are generalization of Vietoris numbers, we find the conditions on coefficient $\left\{a_{k}\right\}$ to characterize the geometric properties of the corresponding analytic function $f(z)=z+\sum_{k=2}^{\infty} a_{k} z^{k}$ in the unit disc $\mathbb{D}$. As an application, we also find geometric properties of generalized Cesàro-type polynomials.
\end{abstract}

\section{Introduction}

Inequalities involving trigonometric sums arise naturally in various problems of pure and applied mathematics. Inequalities that assure nonnegativity or boundedness of partial sums of trigonometric series are of particular interest and applications in various fields. For example, the positivity of trigonometric polynomials are studied in geometric function theory by Gluchoff and Hartmann [1] and Ruscheweyh and Salinas [2]. For a detailed application in signal processing, we refer to the monograph of Dumitrescu [3]. For other applications in this direction, we refer to Dimitrov and Merlo [4], Fernández-Durán [5], and Gasper [6]. The positive trigonometric polynomials played an important role in the proof of Bieberbach conjecture; see [7]. For the applications of positive trigonometric polynomials in Fourier series, approximation theory, function theory, and number theory, we refer to the work of Dimitrov [8] and references therein. For the study of extremal problems, we refer to the dissertation of Révész [9] wherein several applications are outlined.

The problem of finding the behaviour of the coefficients to validate the positivity of trigonometric sum has been dealt by many researchers. Among them, the contributions of Vietoris [10] followed by Koumandos [11] are of interest to the present investigation. Precisely, Vietoris [10] gave sufficient conditions on the coefficient of a general class of sine and cosine sums that ensure their positivity in $(0, \pi)$. For further details in this direction one can refer to [11-13] and the references therein. An account of recent results available in this direction is given in [13] and one of the main results in [13] is as follows.

Theorem 1 (see [13]). Suppose that $\alpha \geq 0, \beta \geq 0$, and $\lambda, \mu \geq 0$ such that $\lambda+\mu \geq 1$ then for $b_{0}=2, b_{1}=1$, and $b_{k}=1 /(k+$ $\alpha)^{\lambda}(k+\beta)^{\mu}, k \geq 2$, we have

$$
\begin{array}{r}
\frac{b_{0}}{2}+\sum_{k=1}^{n} b_{k} \cos k \theta>0, \\
\sum_{k=1}^{n} b_{k} \sin k \theta>0,
\end{array}
$$

for $0<\theta<\pi$ and $n \in \mathbb{N}$.

Using summation by parts, the following corollary of Theorem 1 can be obtained.

Corollary 2. For $\alpha \geq 0, \beta \geq 0$ and $\lambda \geq 0, \mu \geq 0$ such that $\lambda+\mu \geq 1$. If there exists a sequence $\left\{a_{k}\right\}$ of positive real numbers, such that

$$
\begin{gathered}
(k+1+\alpha)^{\lambda}(k+1+\beta)^{\mu} a_{k+1} \leq(k+\alpha)^{\lambda}(k+\beta)^{\mu} a_{k} \\
\leq \cdots \leq(2+\alpha)^{\lambda}(2+\beta)^{\mu} a_{2} \leq a_{1} \leq \frac{a_{0}}{2},
\end{gathered}
$$


then, for $n \in \mathbb{N}$, the following inequalities hold:

$$
\begin{array}{r}
\frac{a_{0}}{2}+\sum_{k=1}^{n} a_{k} \cos k \theta>0 \\
\sum_{k=1}^{n} a_{k} \sin k \theta>0
\end{array}
$$

$$
\text { where } 0<\theta<\pi \text {. }
$$

The main purpose of this note is to use Corollary 2 to find certain geometric properties of analytic functions, in particular univalent functions. Let $\mathscr{A}_{0}$ be the subclass of the class of analytic functions $f \in \mathscr{A}$ with normalized conditions $f(0)=0, f^{\prime}(0)=1$ in the unit disc $\mathbb{D}=\{z \in \mathbb{D},|z|<1\}$. The subclasses of $\mathscr{A}_{0}$ consisting of univalent function are denoted by $\mathcal{S}$. Several subclasses of univalent functions play a prominent role in the theory of univalent functions. For $0 \leq$ $\gamma<1$, let $\mathcal{S}^{*}(\gamma)$ be the family of functions $f$ starlike of order $\gamma$; that is, if $f \in \mathscr{A}_{0}$ satisfies the analytic characterization,

$$
\begin{gathered}
f \in \mathcal{S}^{*}(\gamma) \Longleftrightarrow \\
\operatorname{Re}\left(\frac{z f^{\prime}(z)}{f(z)}\right)>\gamma, \quad \text { for } z \in \mathbb{D} .
\end{gathered}
$$

For $0 \leq \gamma<1$, let $C(\gamma)$ be the family of functions $f$ convex of order $\gamma$; that is, if $f \in \mathscr{A}_{0}$ satisfies the analytic characterization,

$$
\begin{gathered}
f \in \mathscr{C}(\gamma) \Longleftrightarrow \\
\operatorname{Re}\left(1+\frac{z f^{\prime \prime}(z)}{f^{\prime}(z)}\right)>\gamma, \quad \text { for } z \in \mathbb{D} .
\end{gathered}
$$

These two classes are related by the Alexander transform, $f \in \mathscr{C}(\gamma) \Leftrightarrow z f^{\prime} \in \mathcal{S}^{*}(\gamma)$. The usual classes of starlike functions (with respect to origin) and convex functions are denoted, respectively, by $\mathcal{S}^{*}(0) \equiv \mathcal{S}^{*}$ and $\mathscr{C}(0) \equiv \mathscr{C}$. An analytic function $f$ is said to be close-to-convex of order $\gamma$, $(0 \leq \gamma<1)$ with respect to a fixed starlike function $g$ if and only if it satisfies the analytic characterization:

$$
\begin{aligned}
\operatorname{Re} e^{i \eta}\left(\frac{z f^{\prime}(z)}{g(z)}-\mu\right) & >0, \\
& z \in \mathbb{D}, \eta \in\left(-\frac{\pi}{2}, \pi, 2\right), g \in \mathcal{S}^{*} .
\end{aligned}
$$

The family of all close-to-convex function of order $\mu$ with respect to $g \in \mathcal{S}^{*}$ is denoted by $\mathscr{K}_{g}(\mu)$. Further, for $0 \leq \mu<1$, each $f \in \mathscr{K}_{g}(\mu)$ is also univalent in $\mathbb{D}$. The proper inclusion between these classes is given by

$$
\mathscr{C} \varsubsetneqq \mathcal{S}^{*} \varsubsetneqq \mathscr{K} \varsubsetneqq \mathcal{S} .
$$

Another important subclass is the class of typically real functions. A function $f \in \mathscr{A}_{0}$ is typically real if $\operatorname{Im}(z) \operatorname{Im}(f(z)) \geq$ 0 where $z \in \mathbb{D}$. Its class is denoted by $\mathscr{T}$. For several interesting geometric properties of these classes, one can refer to the standard monographs [14-16] on univalent functions.
Remark 3. The functions

$$
z, \frac{z}{1 \pm z}, \frac{z}{1 \pm z^{2}}, \frac{z}{(1 \pm z)^{2}}, \frac{z}{\left(1 \pm z+z^{2}\right)}
$$

are the only nine starlike univalent functions having integer coefficients in $\mathbb{D}$. It will be interesting to find $f$ to be close-toconvex when the corresponding starlike function $g$ takes one of the above forms.

If we take $\eta=0$ and $g(z)=z /(1-z)^{2}$ then $\operatorname{Re}\left((1-z)^{2} f^{\prime}(z)\right)>0$ which implies $z f^{\prime}(z)$ is typically real function. A function $f \in \mathscr{A}_{0}$ is said to be typically real if $\operatorname{Im} f(z) \operatorname{Im}(z)>0$ whenever $\operatorname{Im}(z) \neq 0, z \in \mathbb{D}$. The function $k_{\gamma}(z):=z /(1-z)^{2-2 \gamma}$ is the extremal function for the class of starlike function of order $\gamma$. Note that $k_{0}(z)$ is the wellknown Koebe function and the function $k_{1 / 2}(z)=z /(1-z)$ is the extremal function for the class $\mathscr{C}$. A function $f(z)$ is said to be prestarlike of order $\gamma, 0 \leq \gamma<1$, if $k_{\gamma}(z) * f(z)=$ $z /(1-z)^{2} * f(z) \in \mathcal{S}^{*}(\gamma)$ where “*” is the convolution operator or Hadamard product. This class was introduced by Ruscheweyh [17]. For more details of this class see [18]. Here the Hadamard product or convolution is defined as follows: let $f(z)=\sum_{k=0}^{\infty} a_{k} z^{k}$ and $g(z)=\sum_{k=0}^{\infty} b_{k} z^{k}, z \in \mathbb{D}$. Then,

$$
(f * g)(z)=\sum_{k=0}^{\infty} a_{k} b_{k} z^{k}, \quad z \in \mathbb{D} .
$$

Among all applications of positivity of trigonometric polynomials, the geometric properties of the subclasses of analytic functions are considered in this note. In this direction, Ruscheweyh [19] obtained some coefficient conditions for the class of starlike functions using the classical result of Vietoris [10]. So it would be interesting to find the geometric properties of function $f(z)$ in which Corollary 2 plays a vital role.

\section{Geometric Properties of an Analytic Function}

In this section, we provide conditions on the Taylor coefficients of an analytic function $f$ to guarantee the admissibility of $f$ in subclasses of $\mathcal{S}$, using Corollary 2. The next lemma which is the generalization of [19, Lemma 2] is the crucial ingredient in the proof of the following theorem.

Lemma 4 (see [20, Theorem 3.1]). Let $0 \leq \gamma<1$ and $f \in \mathscr{A}$ be such that $f^{\prime}(z)$ and $f^{\prime}(z)-\gamma(f(z) / z)$ are typically real in $\mathbb{D}$. Further if $\operatorname{Re} f^{\prime}(z)>0$ and $\operatorname{Re}\left(f^{\prime}(z)-\gamma(f(z) / z)\right)>0$, then $f \in \mathcal{S}^{*}(\gamma)$.

Theorem 5. Let $\alpha \geq 0, \beta \geq 0, \lambda \geq 0, \mu \geq 0$ such that $\lambda+\mu \geq 1$; let $\left\{a_{k}\right\}_{k=1}^{\infty}$ be any sequence of positive real numbers such that $a_{1}=1$. Let $\left\{a_{k}\right\}$ satisfy the following conditions:

(1) $(2-\gamma) a_{2} \leq(1-\gamma) a_{1}$.

(2) $(3-\gamma) a_{3} \leq\left(1 /(2+\alpha)^{\lambda}(2+\beta)^{\mu}\right)(2-\gamma) a_{2}$.

(3) $(k+2-\gamma) a_{k+2} \leq(1+1 /(k+\alpha))^{-\lambda}(1+1 /(k+\beta))^{-\mu}(k+$ $1-\gamma) a_{k+1}, \forall k \geq 2$. 
Then, for $0 \leq \gamma<1, f_{n}(z)=z+\sum_{k=1}^{n} a_{k} z^{k}$ and $f(z)=$ $\lim _{n \rightarrow \infty} f_{n}(z)=z+\sum_{k=2}^{\infty} a_{k} z^{k}$ are starlike of order $\gamma$.

Proof. Let $f_{n}(z)=z+\sum_{k=2}^{n} a_{k} z^{k}, z \in \mathbb{D}$ be the partial sum of $f$. Then $f_{n}^{\prime}(z)=1+\sum_{k=1}^{n-1}(k+1) a_{k+1} z^{k}$.

Define

$$
g_{n}(z):=f_{n}^{\prime}(z)-\gamma \frac{f_{n}(z)}{z}=\frac{b_{0}}{2}+\sum_{k=1}^{n-1} b_{k} z^{k}, \quad z \in \mathbb{D}
$$

where $b_{0}=2(1-\gamma)$ and $b_{k}=(k+1-\gamma) a_{k+1}, \forall k \geq 1$. Consider,

$$
\begin{aligned}
& \frac{b_{0}}{2}-b_{1}=(1-\gamma) a_{1}-(2-\gamma) a_{2} \geq 0, \\
& \frac{b_{1}}{(2+\alpha)^{\lambda}(2+\beta)^{\mu}}-b_{2} \\
& =\frac{1}{(2+\alpha)^{\lambda}(2+\beta)^{\mu}}(2-\gamma) a_{2}-(3-\gamma) a_{3} \geq 0 .
\end{aligned}
$$

Now, for $k \geq 2$,

$$
\begin{aligned}
(k & +\alpha)^{\lambda}(k+\beta)^{\mu} b_{k}-(k+1+\alpha)^{\lambda}(k+1+\beta)^{\mu} b_{k+1} \\
& =(k+1+\alpha)^{\lambda}(k+1+\beta)^{\mu} \\
& \times\left[\left(1+\frac{1}{k+\alpha}\right)^{-\lambda}\left(1+\frac{1}{k+\beta}\right)^{-\mu}(k+1-\gamma) a_{k+1}\right. \\
& \left.-(k+2-\gamma) a_{k+2}\right] \geq 0 .
\end{aligned}
$$

So by the given hypothesis, $\left\{b_{k}\right\}$ satisfy the conditions of Corollary 2 which implies $\operatorname{Re} g_{n}(z)>0$ and $\operatorname{Im} g_{n}(z)>0$ if $\operatorname{Im}(z)>0$. By reflection principle $\operatorname{Im} g_{n}(z)<0$ if $\operatorname{Im}(z)<$ 0 . So $g_{n}(z)$ is typically real function. In order to prove the theorem it is remaining to show that $\operatorname{Re} f_{n}^{\prime}(z)>0$ and $f_{n}^{\prime}(z)$ is typically real. In this case $b_{k}=(k+1) a_{k+1}$ and $b_{0}=2$. So such $b_{k}$ also satisfy given hypothesis because $(k+1-\gamma) /(k+2-\gamma)<$ $(k+1) /(k+2)$, for all $k \geq 0$. So $\operatorname{Re} f_{n}^{\prime}(z)>0$ and again using reflection principle we get that $f_{n}^{\prime}(z)$ is typically real in $\mathbb{D}$.

Applying Lemma 4, we get that $f_{n}(z) \in \mathcal{S}^{*}(\gamma)$. Since $\lim _{n \rightarrow \infty} f_{n}(z)=f(z)$ and the family of starlike functions is normal [21, p. 217], we get $f(z)=\lim _{n \rightarrow \infty} f_{n}(z)$ is also starlike of order $\gamma$.

Remark 6. If $\gamma=0$ in Theorem 5, then we get $\operatorname{Re}\left(f_{n}^{\prime}(z)\right)>0$ which implies $f_{n}(z)$ is close-to-convex with respect to $z$ and $f_{n}^{\prime}(z)$ is typically real also and with

$$
\begin{aligned}
\operatorname{Re}(1-z) f_{n}^{\prime}(z)= & \operatorname{Re}(1-z) \operatorname{Re} f_{n}^{\prime}(z) \\
& +\operatorname{Im}(z) \operatorname{Im} f_{n}^{\prime}(z)>0
\end{aligned}
$$

this yields $f_{n}(z)$ that is close-to-convex with respect to starlike function $z /(1-z)$.
Example 7. Consider the sequence $\left\{a_{k}\right\}$ as $a_{1}=1, a_{2}=1 / 2$, and $a_{k}=1 / k^{2}$ for $k \geq 3$; then by Theorem 5 , the function

$$
f(z)=z+\frac{z^{2}}{2}+\sum_{k=3}^{\infty} \frac{z^{k}}{k^{2}}, \quad z \in \mathbb{D}
$$

is starlike univalent. But [22, Theorem 2.1] fails to include this function. Hence Theorem 5 is better than [22, Theorem 2.1] in the sense that it is likely to include more cases.

By proving that $z f_{n}^{\prime}(z)$ is typically real function in the similar fashion, we obtain the next result.

Theorem 8. Let $\alpha \geq 0, \beta \geq 0$ and $\lambda \geq 0, \mu \geq 0$ such that $\lambda+\mu \geq 1$; let $\left\{a_{k}\right\}_{k=1}^{\infty}$ be any sequence of positive real numbers such that $a_{1}=1$, if $\left\{a_{k}\right\}$ satisfy the following conditions:

$$
\begin{aligned}
(k & +1+\alpha)^{\lambda}(k+1+\beta)^{\mu}(k+1) a_{k+1} \\
& \leq(k+\alpha)^{\lambda}(k+\beta)^{\mu} k a_{k} \leq \cdots \\
& \leq(2+\alpha)^{\lambda}(2+\beta)^{\mu} 2 a_{2} \leq 1, \quad \text { for } k \geq 2 .
\end{aligned}
$$

Then $f_{n}(z)=z+\sum_{k=2}^{n} a_{k} z^{k}$ and $f(z)=z+\sum_{k=2}^{\infty} a_{k} z^{k}$ are close-to-convex with respect to starlike function $z /\left(1-z^{2}\right)$.

Note that Theorem 8 provides close-to-convexity of $f$ with respect to the function $z /\left(1-z^{2}\right)$. Results for the close-to-convexity of $f$ with respect to other four starlike functions given in Remark 3 are of considerable interest, and the authors have considered some of these cases separately elsewhere. The next result provides the coefficient conditions for $f$ to be in the class of prestarlike functions of order $\gamma$, $0 \leq \gamma<1$.

Theorem 9. Let $\alpha \geq 0, \beta \geq 0, \lambda \geq 0, \mu \geq 0$ such that $\lambda+\mu \geq 1$; let $\left\{a_{k}\right\}_{k=1}^{\infty}$ be any sequence of positive real numbers such that $a_{1}=1$. Let $\left\{a_{k}\right\}$ satisfy the following conditions:

(1) $(2+\alpha)^{\lambda}(2+\beta)^{\mu}(3-\gamma)(3-2 \gamma) a_{3} \leq 2(2-\gamma) a_{2} \leq a_{1}$.

(2) $(k+1+\alpha)^{\lambda}(k+1+\beta)^{\mu}(k+2-\gamma)(k+2-2 \gamma) a_{k+2} \leq$ $(k+\alpha)^{\lambda}(k+\beta)^{\mu}(k+1-\gamma)(k+1) a_{k+1}, \forall k \geq 2$.

Then for $0 \leq \gamma<1, f_{n}(z)=z+\sum_{k=2}^{n} a_{k} z^{k}$ is prestarlike of order $\gamma$. Moreover, $f(z)=z+\sum_{k=2}^{\infty} a_{k} z^{k}$ is prestarlike of order $\gamma$.

Proof. Let $g_{n}(z):=f_{n}(z) * z /(1-z)^{2-2 \gamma}, z \in \mathbb{D}, 0 \leq \gamma<1$. To prove required theorem, it is sufficient to prove that $g_{n}(z) \in$ $\mathcal{S}^{*}(\gamma)$ :

$$
\begin{aligned}
g_{n}(z) & =f_{n}(z) * \frac{z}{(1-z)^{2-2 \gamma}} \\
& =z+\sum_{k=2}^{n} \frac{(2-2 \gamma)_{k-1}}{(k-1) !} a_{k} z^{k}, \quad z \in \mathbb{D} .
\end{aligned}
$$

We prove that $g_{n}(z)$ satisfy the conditions of Lemma 4 . For this, define

$$
h_{n}(z):=g_{n}^{\prime}(z)-\gamma \frac{g_{n}(z)}{z}=\frac{b_{0}}{2}+\sum_{k=1}^{n-1} b_{k} z^{k}, \quad z \in \mathbb{D},
$$


where $b_{0}=2(1-\gamma)$ and $b_{k}=(k+1-\gamma)\left((2-2 \gamma)_{k} / k !\right) a_{k+1}$ for $k \geq$ 1. Using simple calculations, along with the hypothesis, $\left\{b_{k}\right\}$ satisfy the conditions of Corollary 2 . Continuing the same argument as earlier, we get the desired result.

Remark 10 . Note that $\mathscr{R}(1 / 2)=\mathcal{S}^{*}(1 / 2)$. It can be easily verified that all the conditions of Theorem 9 for $\mathscr{R}(1 / 2)$ coincide with the conditions of Theorem 5 for $\mathcal{S}^{*}(1 / 2)$. diate.

For $\gamma=0, \mathscr{R}^{*}(0) \equiv \mathscr{C}$ and the following result is imme-

Corollary 11. For $\alpha \geq 0, \beta \geq 0, \lambda \geq 0, \mu \geq 0$ such that $\lambda+\mu \geq 1$, let $\left\{a_{k}\right\}_{k=1}^{\infty}$ be any sequence of positive real numbers such that $a_{1}=1$. Let $\left\{a_{k}\right\}$ satisfy the following condition:

$$
\begin{gathered}
(k+1+\alpha)^{\lambda}(k+1+\beta)^{\mu}(k+2)^{2} a_{k+2} \\
\leq(k+\alpha)^{\lambda}(k+\beta)^{\mu}(k+1)^{2} a_{k+1} \leq \cdots \\
\leq(2+\alpha)^{\lambda}(2+\beta)^{\mu} 9 a_{3} \leq 4 a_{2} \leq a_{1} .
\end{gathered}
$$

Then $f_{n}(z)=z+\sum_{k=2}^{n} a_{k} z^{k}$ is convex function. In particular $f(z)=z+\sum_{k=2}^{\infty} a_{k} z^{k}$ is convex univalent.

Example 12. Let $f(z)=z+z^{2} / 4+\sum_{k=3}^{\infty}\left(z^{k} /(k-1+\alpha)^{\lambda}(k-\right.$ $1+\beta)^{\mu} k^{2}$ ) is convex univalent.

In particular if $\alpha=\beta=1$ and $\lambda=\mu=1 / 2$, we get that $z+z^{2} / 4+\sum_{k=3}^{\infty}\left(z^{k} / k^{3}\right)$ is convex.

\section{Application to Cesàro Mean of Type $(b-1, c)$}

The $n$th Cesàro mean of type $(b-1, c)$ of $f(z) \in \mathscr{A}_{0}$ is given by

$$
\begin{aligned}
s_{n}^{(b-1, c)}(z, f) & :=z+\sum_{k=2}^{n} \frac{B_{n-k}}{B_{n-1}} a_{k} z^{k} \\
& =s_{n}^{(b-1, c)}(z) * f(z), \quad n \in \mathbb{N},
\end{aligned}
$$

where $b$ and $c$ are real numbers such that $b+1>c>0$ and $B_{0}=1$ and $B_{k}=((1+b-c) / b)\left((b)_{k} /(c)_{k}\right)$ for $k \geq 1$. Here by $(\alpha)_{k}, k \geq \mathbb{N}$, which is the well-known Pochhammer symbol, we mean the following:

$$
(\alpha)_{k}=\alpha(\alpha+1)_{k-1} \quad \text { with }(\alpha)_{0}=1 .
$$

For $b=1+\delta$ and $c=1$, it follows that

$$
\begin{aligned}
s_{n}^{(\delta, 1)}(f, z) & =s_{n}^{\delta}(f, z) \\
& =z+\sum_{k=2}^{n} \frac{(1+\delta)_{n-k}}{(n-k) !} \frac{(n-1) !}{(1+\delta)_{n-1}} a_{k} z^{k},
\end{aligned}
$$

which is the Cesàro mean of order $\delta$ for $\delta>-1$. Since (19) is one type of generalization of the well-known Cesàro mean [23], we call these Cesàro means of type $(b-1 ; c)$ as generalized Cesàro operators. The coefficients given in (19) were considered in [13] while finding positivity of trigonometric polynomials. Using (19), generalized Cesàro averaging operators were studied in [24] which are generalization of the Cesàro operator given by Stempak [25]. The geometric properties of $s_{n}^{\delta}(z)$ are well-known. For details, see [23, 26, 27]. Lewis [28] proved that $s_{n}^{\delta}(z)$ is close-to-convex and hence univalent for $\delta \geq 1$. Ruscheweyh [23] proved that it is prestarlike of order $(3-\delta) / 2$. Hence it would be interesting to see if the geometric properties of $s_{n}^{\delta}(z)$ can be extended to $s_{n}^{(b-1, c)}(f, z)$. Such investigations are possible by various known methods in geometric function theory. In particular, the positivity techniques used in Koumandos [11] or Mondal and Swaminathan [20] can be applied to $s_{n}^{(b-1, c)}(z)$ as well. However, in view of Example 7, we are interested in using the results available in Section 2 to obtain the geometric properties of $s_{n}^{(b-1, c)}(z)$.

Theorem 13. Let $\left\{a_{k}\right\}$ be any sequence of positive real numbers such that $a_{1}=1$ and $(b+n-2) a_{1} \geq 2(c+n-2) a_{2}$. Let $b \geq c>0$, $0 \leq \alpha \leq 6 /(\lambda+4), 0 \leq \beta \leq 6 /(\mu+4)$, and $\lambda, \mu \geq 0$ such that $\lambda+\mu \geq 1$ and $1 \leq \lambda+\mu<2$ and satisfy the following conditions:

(i) $(2-\alpha \lambda)(2-\beta \mu)(b+n-3) a_{2} \geq 2^{\lambda+\mu+1}(c+n-3) 3 a_{3}$.

(ii) $(k-1+\alpha-\lambda)(k-1+\beta-\mu)(b+n-k-1) k a_{k} \geq$ $(k-1+\alpha)(k-1+\beta)(c+n-k-1)(k+1) a_{k+1}$ for $3 \leq k \leq n-3$.

(iii) $(n-2+\alpha-\lambda)(n-2+\beta-\mu)(1+b-c)(n-1) a_{n-1} \geq$ $(n-2+\alpha)(n-2+\beta) c n a_{n}$.

Then $s_{n}^{(b-1, c)}(f, z)$ is close-to-convex with respect to $z$ and $z /(1-$ $z)$ where $f(z)=z+\sum_{k=2}^{\infty} a_{k} z^{k}$. Further for the same condition $s_{n}^{(b-1, c)}(f, z)$ is starlike univalent.

Proof. Let $s_{n}^{(b-1, c)}(f, z)=z+\sum_{k=2}^{n}\left(B_{n-k} / B_{n-1}\right) a_{k} z^{k}$. Then,

$$
s_{n}^{(b-1, c)}(f, z)^{\prime}=1+\sum_{k=1}^{n-1} \frac{B_{n-k-1}}{B_{n-1}}(k+1) a_{k+1} z^{k} .
$$

For $0 \leq r<1$ and $0 \leq \theta \leq 2 \pi$,

$$
\begin{aligned}
& \operatorname{Re} s_{n}^{(b-1, c)}(f, z)^{\prime}=\frac{b_{0}}{2}+\sum_{k=1}^{n-1} b_{k} r^{k} \cos k \theta \\
& \operatorname{Im} s_{n}^{(b-1, c)}(f, z)^{\prime}=\sum_{k=1}^{n-1} b_{k} r^{k} \sin k \theta
\end{aligned}
$$

where $b_{0}=2$ and $b_{k}=\left(B_{n-k-1} / B_{n-1}\right)(k+1) a_{k+1}$ for $k \geq 1$. Hence $b_{k}$ and $b_{k+1}$ can be related as follows:

$$
\begin{aligned}
& b_{k+1}=\frac{(c+n-k-2)(k+2) a_{k+2}}{(b+n-k-2)(k+1) a_{k+1}} b_{k}, \\
& b_{n-1}=\frac{c}{(1+b-c)} \frac{n a_{n}}{(n-1) a_{n-1}} b_{n-2} .
\end{aligned}
$$


For the sequence $\left\{b_{k}\right\}$, our aim is to prove that $b_{0} / 2+$ $\sum_{k=1}^{n-1} b_{k} r^{k} \cos k \theta>0$ and $\sum_{k=1}^{n-1} b_{k} r^{k} \sin k \theta>0$. Note that

$$
\begin{aligned}
\frac{b_{0}}{2} & -b_{1} \\
& =\frac{1}{(b+n-2)}\left[(b+n-2) a_{1}-2(c+n-2) a_{2}\right] \\
& \geq 0 .
\end{aligned}
$$

For a given $\alpha$ and $\beta$, we can easily get

$$
\begin{aligned}
& \frac{1}{(2+\alpha)^{\lambda}(2+\beta)^{\mu}}=\frac{1}{2^{\lambda+\mu}}\left(1+\frac{\alpha}{2}\right)^{-\lambda}\left(1+\frac{\beta}{2}\right)^{-\mu} \\
& =\frac{1}{2^{\lambda+\mu}}\left(1-\frac{\alpha \lambda}{2}+\frac{\lambda(\lambda+1)}{2 !} \frac{\alpha^{2}}{2^{2}}\right. \\
& -\frac{\lambda(\lambda+1)(\lambda+2)}{3 !} \frac{\alpha^{3}}{2^{3}} \\
& \left.+\frac{\lambda(\lambda+1)(\lambda+2)(\lambda+3)}{4 !} \frac{\alpha^{4}}{2^{4}}-\cdots\right) \times\left(1-\frac{\beta \mu}{2}\right. \\
& +\frac{\mu(\mu+1)}{2 !} \frac{\beta^{2}}{2^{2}}-\frac{\mu(\mu+1)(\mu+2)}{3 !} \frac{\beta^{3}}{2^{3}} \\
& \left.+\frac{\mu(\mu+1)(\mu+2)(\mu+3)}{4 !} \frac{\beta^{4}}{2^{4}}-\cdots\right) \\
& =\frac{1}{2^{\lambda+\mu+2}}[(2-\alpha \lambda) \\
& +\lambda(\lambda+1) \frac{\alpha^{2}}{2^{2}}\left(1-\frac{(\lambda+2)}{6} \alpha\right) \\
& +\frac{\lambda(\lambda+1)(\lambda+2)(\lambda+3)}{3 \cdot 2^{6}} \alpha^{4}\left(1-\frac{(\lambda+4)}{10} \alpha\right) \\
& +\cdots] \times[(2-\beta \mu) \\
& +\mu(\mu+1) \frac{\beta^{2}}{2^{2}}\left(1-\frac{(\mu+2)}{6} \beta\right) \\
& +\frac{\mu(\mu+1)(\mu+2)(\mu+3)}{3 \cdot 2^{6}} \beta^{4}\left(1-\frac{\mu+4}{10} \beta\right) \\
& +\cdots] \geq \frac{(2-\alpha \lambda)(2-\beta \mu)}{2^{\lambda+\mu+2}} \\
& \text { if } 0 \leq \alpha \leq \frac{6}{\lambda+2}, 0 \leq \beta \leq \frac{6}{\mu+2} \text {. }
\end{aligned}
$$

Hence we see that

$$
\begin{aligned}
& \frac{b_{1}}{(2+\alpha)^{\lambda}(2+\beta)^{\mu}}-b_{2} \\
& \geq \frac{(2-\alpha \lambda)(2-\beta \mu) b_{1}}{2^{\lambda+\mu+2}}-\frac{(c+n-3) 3 a_{3}}{(b+n-3) 2 a_{2}} b_{1} \geq 0 .
\end{aligned}
$$

For the other condition $(k+\alpha)^{\lambda}(k+\beta)^{\mu} b_{k} \geq(k+1+\alpha)^{\lambda}(k+$ $1+\beta)^{\mu} b_{k+1}$ to be satisfied, first we find

$$
\begin{aligned}
& {\left[1+\frac{1}{k+\alpha}\right]^{-\lambda}\left[1+\frac{1}{k+\beta}\right]^{-\mu}=\left[1-\frac{\lambda}{k+\alpha}\right.} \\
& +\frac{\lambda(\lambda+1)}{2 !(k+\alpha)^{2}}\left(1-\frac{(2+\lambda)}{3(k+\alpha)}\right) \\
& \left.+\frac{\lambda(\lambda+1)(\lambda+2)(\lambda+3)}{4 !(k+\alpha)^{4}}\left(1-\frac{\lambda+4}{5(k+\alpha)}\right)+\cdots\right] \\
& \times\left[1-\frac{\mu}{k+\beta}+\frac{\mu(\mu+1)}{2 !(k+\beta)^{2}}\left(1-\frac{(2+\mu)}{3(k+\beta)}\right)\right. \\
& +\frac{\mu(\mu+1)(\mu+2)(\mu+3)}{4 !(k+\beta)^{4}}\left(1-\frac{\mu+4}{5(k+\beta)}\right) \\
& +\cdots] \geq\left(1-\frac{\lambda}{k+\alpha}\right) \cdot\left(1-\frac{\mu}{k+\beta}\right), \\
& \quad \text { if } \frac{2+\lambda}{3(k+\alpha)} \leq 1, \frac{2+\mu}{3(k+\beta)} \leq 1 \text { for } k \geq 2 .
\end{aligned}
$$

Clearly,

$$
\begin{aligned}
& \left(1+\frac{1}{k+\alpha}\right)^{-\lambda}\left(1+\frac{1}{k+\beta}\right)^{-\mu} b_{k}-b_{k+1} \geq(1 \\
& \left.-\frac{\lambda}{k+\alpha}\right)\left(1-\frac{\mu}{k+\beta}\right) b_{k} \\
& -\frac{(c+n-k-2)(k+2) a_{k+2}}{(b+n-k-2)(k+1) a_{k+1}} b_{k} \\
& =b_{k-1}\left[\left(1-\frac{\lambda}{k-1+\alpha}\right)\left(1-\frac{\mu}{k-1+\beta}\right)\right. \\
& \left.-\frac{(c+n-k-1)(k+1) a_{k+1}}{(b+n-k-1) k a_{k}}\right] \geq 0 \\
& \quad \text { for } 3 \leq k \leq n-2 .
\end{aligned}
$$

For $k=n-2$, consider

$$
\begin{aligned}
& \frac{1}{(1+1 /(n-2+\alpha))^{\lambda}(1+1 /(n-2+\beta))^{\mu}} b_{n-2}-b_{n-1} \\
& \geq\left(1-\frac{\lambda}{n-2+\alpha}\right)\left(1-\frac{\mu}{n-2+\beta}\right) \\
& \cdot \frac{(1+b-c)}{c}(n-1) a_{n-1}-n a_{n} \geq 0 .
\end{aligned}
$$

We proved that $b_{0} / 2+\sum_{k=1}^{n-1} b_{k} \cos k \theta>0$ and $\sum_{k=1}^{n-1} b_{k} \sin k \theta>$ 0 for $0<\theta<\pi$. By the minimum principle for harmonic functions, $b_{0} / 2+\sum_{k=1}^{n-1} b_{k} r^{k} \cos k \theta>0,0 \leq r<1$ and $0<\theta<\pi$ and $\sum_{k=1}^{n-1} b_{k} r^{k} \sin k \theta>0$ for $0<\theta<\pi$ and 
$0 \leq r<1$. Using reflection principle, $\sum_{k=1}^{n-1} b_{k} r^{k} \sin k \theta<0$ for $\pi<\theta<2 \pi$ and $0 \leq r<1$. Note that $s_{n}^{(b-1, c)}(f, z)$ is close-to-convex with respect to $z$ if $\operatorname{Re} s_{n}^{(b-1, c)}(f, z)>0$ and $s_{n}^{(b-1, c)}(f, z)$ is close-to-convex with respect to $z /(1-z)$ if $\operatorname{Re}\left[(1-z) s_{n}^{(b-1, c)}(f, z)^{\prime}\right]>0$. Now

$$
\begin{aligned}
\operatorname{Re} & {\left[(1-z) s_{n}^{(b-1, c)}(f, z)^{\prime}\right] } \\
= & \operatorname{Re}(1-z) \operatorname{Re}\left(s_{n}^{(b-1, c)}(f, z)^{\prime}\right) \\
& -\operatorname{Im}(1-z) \operatorname{Im}\left(s_{n}^{(b-1, c)}(f, z)^{\prime}\right) \\
= & \operatorname{Re}(1-z) \operatorname{Re}\left(s_{n}^{(b-1, c)}(f, z)^{\prime}\right) \\
& +\operatorname{Im}(z) \operatorname{Im}\left(s_{n}^{(b-1, c)}(f, z)^{\prime}\right)>0 .
\end{aligned}
$$

For $b=1+\delta, c=1$, Theorem 13 leads to the following example.

Example 14. Let $\lambda \geq 0, \mu \geq 0$ such that $1 \leq \lambda+\mu<2$, $0 \leq \alpha \leq 6 /(\lambda+4)$, and $0 \leq \beta \leq 6 /(\beta+4)$; then

$$
\begin{aligned}
\delta \geq & \max _{n \geq 1}\left\{0,(n-2)\left(\frac{2^{\lambda+\mu+2}}{(2-\alpha \lambda)(2-\beta \mu)}-1\right),(n-3)\right. \\
& \left.\cdot\left(\frac{2(\lambda+\mu)+\alpha \mu+\beta \lambda+\lambda \mu}{(2+\alpha-\lambda)(2+\beta-\mu)}\right)\right\} .
\end{aligned}
$$

Then $s_{n}^{\delta}(-\log (1-z), z)$ is close-to-convex with respect to $z$ and $z /(1-z)$. Further for the same condition it is also starlike univalent.

Remark 15. If we take $\alpha=\beta=1$ and $\lambda=\mu=1 / 2$ then, for $1 \leq n \leq 3, s_{n}^{\delta}(-\log (1-z), z)$ is close-to-convex with respect to $z$ and $z /(1-z)$ for $\delta \geq \delta^{\prime}$ where $0<\delta^{\prime}<3$. This conclusion cannot be obtained from [20, Corollary 4.2].

Theorem 16. Let $\left\{a_{k}\right\}$ be a sequence of positive real numbers with $a_{1}=1$ and satisfy the hypothesis of Theorem 13. Then $s_{n}^{(b-1, c)}(f, z) \in \mathscr{R}(\gamma), \gamma \geq 0$ where

$$
\gamma \leq 1-\frac{(c+n-2)}{(b+n-2)} 2 a_{2},
$$

$\mathscr{R}(\gamma)=\left\{f \in \mathscr{A}: \operatorname{Re} f^{\prime}(z)>\gamma\right\}$ and $f(z)=z+\sum_{k=2}^{\infty} a_{k} z^{k}$, $z \in \mathbb{D}$.

Proof. Let $s_{n}^{(b-1, c)}(f, z)=z+\sum_{k=2}^{n}\left(B_{n-k} / B_{n-1}\right) a_{k} z^{k}$ where $B_{0}=$ 1 and $B_{k}=\left((b)_{k} /(c)_{k}\right)((1+b-c) / b), k \geq 1$ :

$$
s_{n}^{(b-1, c)}(f, z)^{\prime}=1+\sum_{k=1}^{n-1} \frac{B_{n-k-1}}{B_{n-1}}(k+1) a_{k+1} z^{k} .
$$

We consider

$$
\frac{s_{n}^{(b-1, c)}(f, z)^{\prime}-\gamma}{1-\gamma}=\frac{b_{0}}{2}+\sum_{k=1}^{n-1} b_{k} z^{k},
$$

where $b_{0}=2$ and $b_{k}=B_{n-k-1} / B_{n-1} \cdot(k+1) a_{k+1} /(1-\gamma)$ for $k \geq 1$. Then $b_{k}$ and $b_{k+1}$ are related by

$$
b_{k+1}=\frac{(c+n-k-2)(k+2) a_{k+2}}{(b+n-k-2)(k+1) a_{k+1}} b_{k}
$$

for $1 \leq k \leq n-3$,

and, for $k=n-2$,

$$
b_{n-1}=\frac{(1+b-c)}{c} \frac{(n-1) a_{n-1}}{n a_{n}} b_{n-2} .
$$

Using hypothesis, we can easily get

$$
\frac{b_{0}}{2}-b_{1}=1-\left(\frac{c+n-2}{b+n-2}\right) \frac{2 a_{2}}{1-\gamma} \geq 0 .
$$

The relation between the coefficients $b_{k}$ and $b_{k+1}$ is the same as in the Theorem 13. So such $b_{k}$ also satisfy the conditions of Theorem 13 and from Corollary 2 we have the required result that

$$
\frac{b_{0}}{2}+\sum_{k=1}^{n-1} b_{k} \cos k \theta>0 \quad \text { for } 0<\theta<\pi
$$

From the minimum principle for harmonic functions for $0 \leq$ $r<1$ and $0<\theta<2 \pi$ we have

$$
\operatorname{Re}\left(\frac{s_{n}^{(b-1, c)}(f, z)^{\prime}-\gamma}{1-\gamma}\right)=\frac{b_{0}}{2}+\sum_{k=1}^{n-1} b_{k} r^{k} \cos k \theta>0 .
$$

So, $s_{n}^{(b-1, c)}(f, z) \in \mathscr{R}(\gamma)$.

It can be clearly seen that, for $\gamma=0$, Theorem 16 coincides with Theorem 13 for the case $g(z)=z$.

Theorem 17. Let $\left\{a_{k}\right\}_{k=1}^{\infty}$ be a sequence of positive real numbers such that $a_{1}=1$. If for $\lambda \geq 0, \mu \geq 0$ such that $1 \leq \lambda+\mu<2$ and $0 \leq \alpha \leq 6 /(\lambda+4), 0 \leq \beta \leq 6 /(\mu+4), a_{k}$ satisfy the following conditions:

(1) $(3-2 \lambda-2 \mu)(b+n-2) a_{1} \geq(5-2 \lambda-2 \mu)(c+n-2) a_{2}$.

(2) $(2-\alpha \lambda)(2-\beta \mu)(5-2 \lambda-2 \mu)(b+n-3) a_{2} \geq 2^{\lambda+\mu+2}(7-$ $2 \lambda-2 \mu)(c+n-3) a_{3}$.

(3) $(2 k+1-2 \lambda-2 \mu)(k-1+\alpha-\lambda)(k-1+\beta-\mu)(b+$ $n-k-1) a_{k} \geq(2 k+3-2 \lambda-2 \mu)(k-1+\alpha)(k-1+$ $\beta)(c+n-k-1) a_{k+1}$ for $3 \leq k \leq n-2$.

(4) $(n-2+\alpha-\lambda)(n-2+\beta-\mu)(2 n+1-2 \lambda-2 \mu)(1+b-c) a_{n-1} \geq$ $(n-2+\alpha)(n-2+\beta)(2 n+3-2 \lambda-2 \mu) c a_{n}$.

Then, $s_{n}^{(b-1, c)}(f, z) \in \mathcal{S}^{*}(\lambda+\mu-1 / 2)$, where $f(z)=z+$ $\sum_{k=2}^{\infty} a_{k} z^{k}, z \in \mathbb{D}$.

Proof. $s_{n}^{(b-1, c)}(f, z)=z+\sum_{k=2}^{n}\left(B_{n-k} / B_{n-1}\right) a_{k} z^{k}=b_{1} z+$ $\sum_{k=2}^{n} b_{k} z^{k}$, where $b_{1}=1$ and $b_{k}=\left(B_{n-k} / B_{n-1}\right) a_{k}$ for $k \geq 2$. Then,

$$
b_{k+1}=\left(\frac{c+n-k-1}{b+n-k-1}\right) \frac{a_{k+1}}{a_{k}} b_{k}, \quad \text { for } 2 \leq k \leq n-2,
$$


and for $k=n-1, b_{n}=(c /(1+b-c))\left(a_{n} / a_{n-1}\right) b_{n-1}$. It is enough to prove that $\left\{b_{k}\right\}$ satisfy the conditions of Theorem 5 . For the sake of convenience we substitute $\gamma=\lambda+\mu-1 / 2$. By a simple calculation we can get that $(1-\gamma) b_{1}-(2-\gamma) b_{2} \geq 0$. Now

$$
\begin{gathered}
\frac{1}{(2+\alpha)^{\lambda}(2+\beta)^{\mu}}(2-\gamma) b_{2}-(3-\gamma) b_{3} \\
\geq \frac{(2-\alpha \lambda)(2-\beta \mu)}{2^{\lambda+\mu+2}}(5-2 \lambda-2 \mu) b_{2} \\
-(7-2 \lambda-2 \mu) \frac{(c+n-3)}{(b+n-3)} \frac{a_{3}}{a_{2}} b_{2} \geq 0 .
\end{gathered}
$$

Now, for $2 \leq k \leq n-3$,

$$
\begin{aligned}
& \left(1+\frac{1}{k+\alpha}\right)^{-\lambda}\left(1+\frac{1}{k+\beta}\right)^{-\mu}(k+1-\gamma) b_{k+1} \\
& -(k+2-\gamma) b_{k+2} \geq\left(1-\frac{\lambda}{k+\alpha}\right)\left(1-\frac{\mu}{k+\beta}\right) \\
& \cdot(2 k+3-2 \lambda-2 \mu) b_{k+1}-(2 k+5-2 \lambda-2 \mu) \\
& \cdot\left(\frac{c+n-k-2}{b+n-k-2}\right) \frac{a_{k+2}}{a_{k+1}} b_{k+1} \geq 0
\end{aligned}
$$

And, for $k=n-2$, using the hypothesis, we obtain,

$$
\begin{aligned}
& \left(1+\frac{1}{n-2+\alpha}\right)^{-\lambda}\left(1+\frac{1}{n-2+\beta}\right)^{-\mu} \\
& \cdot(2 n+1-2 \lambda-2 \mu) b_{n-1}-(2 n+3-2 \lambda-2 \mu) b_{n}
\end{aligned}
$$

$\geq 0$.

From Theorem 5 the desired result follows.

Theorem 18. Let $b \geq c>0,0 \leq \alpha \leq 6 /(\lambda+4), 0 \leq \beta \leq$ $6 /(\mu+4)$ and $\lambda, \mu \geq 0$ such that $1 \leq \lambda+\mu<2$ and satisfies the following conditions:

(1) $2(2-\gamma)(c+n-2) \leq(b+n-2)$,

(2) $(2+\alpha)^{\lambda}(2+\beta)^{\mu}(3-\gamma)(3-2 \gamma)(c+n-3) \leq 2(2-\gamma)(b+$ $n-3)$,

(3) $(k+1+\alpha)^{\lambda}(k+1+\beta)^{\mu}(k+2-\gamma)(k+2-2 \gamma)(c+n-k-2) \leq$ $(k+\alpha)^{\lambda}(k+\beta)^{\mu}(k+1-\gamma)(k+1)(b+n-k-2)$ for $2 \leq k \leq n-3$,

(4) $(n-1+\alpha)^{\lambda}(n-1+\beta)^{\mu}(n-\gamma)(n-2 \gamma) c \leq(n-2+$ $\alpha)^{\lambda}(n-2+\beta)^{\mu}(n-1-\gamma)(n-1)(1+b-c)$.

Then $s_{n}^{(b-1, c)}(z)$ is prestarlike of order $\gamma$, where $0 \leq \gamma<1$.

Proof. It is given that $s_{n}^{(b-1, c)}(z)=z+\sum_{k=2}^{n}\left(B_{n-k} / B_{n-1}\right) z^{k}=$ $z+\sum_{k=2}^{n} a_{k} z^{k}, z \in \mathbb{D}$.

Then using $a_{k}=B_{n-k} / B_{n-1}$ for $k \geq 1$ in Theorem 5 and following the same procedure the result can be proved.
If $\gamma=0$ then $s_{n}^{(b-1, c)}(z) \in \mathscr{R}^{*}(0)=\mathscr{C}$. Further if we substitute $b=1+\delta$ and $c=1$ in Theorem 18 , we have the following example.

Example 19. If $\alpha, \beta, \lambda$, and $\mu$ satisfy the conditions of Theorem 18 and if

$$
\begin{aligned}
\delta \geq & \max \{(n-1)(3-2 \gamma),(n-2) \\
& \cdot\left(\frac{(2+\alpha)^{\lambda}(2+\beta)^{\mu}(3-\gamma)(3-2 \gamma)}{2(2-\gamma)}-1\right),(n-3) \\
& \left.\cdot\left(\frac{(3+\alpha)^{\lambda}(3+\beta)^{\mu}(4-\gamma)(4-2 \gamma)}{(2+\alpha)^{\lambda}(2+\beta)^{\mu}(3-\gamma) 3}-1\right)\right\} .
\end{aligned}
$$

Then $s_{n}^{\delta}(z)$ is prestarlike of order $\gamma$, where $\gamma \in[0,1)$.

It can be noted that if we take $\alpha=\beta=0$ and $\lambda+\mu=1$ in Example 19, then, for $\delta \geq(n-1)(3-2 \gamma), s_{n}^{\delta}(z) \in \mathscr{R}^{*}(\gamma)$. Similar type of result had been found in [23, Theorem 1]. From [26, Theorem 2.1], we deduce the following corollary.

Corollary 20. If $\alpha, \beta, \lambda, \mu$, and $\gamma$ satisfy the hypothesis of Theorem 18 then for $b \geq c, s_{n}^{(b-1, c)} \in \mathscr{R}^{*}(\gamma)$. Then for any $z g \in \mathscr{K}(\gamma) \Rightarrow g *\left(s_{n}^{(b-1, c)}(z)\right)^{\prime}$ is zero free in $\mathbb{D}$.

The following Lemma, which is the extension of the wellknown Polya-Schoenberg Theorem, is ingredient to our next result.

Lemma 21 (see [17, p. 499]). If $f \in \mathscr{K}(\gamma), g \in \mathscr{R}^{*}(\gamma), 0 \leq$ $\gamma<1$ then $f * g \in \mathscr{K}(\gamma)$.

Clearly, $s_{n}^{(b-1, c)}(g, z) \in \mathscr{K}(\gamma)$ if $g \in \mathscr{K}(\gamma)$.

Theorem 22. Let $\left\{a_{k}\right\}$ be a sequence of positive real numbers such that $a_{1}=1$. Then, for $0 \leq \alpha \leq 6 /(\lambda+4), 0 \leq \beta \leq 6 /(\mu+4)$ and $\lambda \geq 0, \mu \geq 0$ such that $1 \leq \lambda+\mu<2$, if $\left\{a_{k}\right\}$ satisfy the following conditions:

(1) $(2-\alpha \lambda)(2-\beta \mu)(b+n-2) a_{1} \geq(c+n-2) 2^{\lambda+\mu+3} a_{2}$.

(2) $k(k+\alpha-\lambda)(k+\beta-\mu)(b+n-k-1) a_{k} \geq(k+\alpha)(k+$ $\beta)(c+n-k-1)(k+1) a_{k+1}$, for all $2 \leq k \leq n-2$.

(3) $(n-1+\alpha-\lambda)(n-1+\beta-\mu)(1+b-c)(n-1) a_{n-1} \geq$ $c(n-1+\alpha)(n-1+\beta) n a_{n}$.

Then $s_{n}^{(b-1, c)}(f, z)$ is close-to-convex with respect to starlike function $z /\left(1-z^{2}\right)$ where $f(z)=z+\sum_{k=2}^{\infty} a_{k} z^{k}, z \in \mathbb{D}$.

Proof. $s_{n}^{(b-1, c)}(f, z)=z+\sum_{k=2}^{n}\left(B_{n-k} / B_{n-1}\right) a_{k} z^{k}$ is close-toconvex with respect to $z /\left(1-z^{2}\right)$ if $z s_{n}^{(b-1, c)}(f, z)^{\prime}$ is typically real function. Consider

$$
z s_{n}^{(b-1, c)}(f, z)^{\prime}=z+\sum_{k=2}^{n} \frac{B_{n-k}}{B_{n-1}} k a_{k} z^{k}=b_{1} z+\sum_{k=2}^{n} b_{k} z^{k}
$$


where $b_{1}=1$ and $b_{k}=\left(B_{n-k} / B_{n-1}\right) k a_{k}$ for $k \geq 2$. Clearly

$$
\begin{gathered}
b_{k+1}=\frac{B_{n-k-1}}{B_{n-1}}(k+1) a_{k+1} \\
\begin{cases}b_{k+1}=\frac{(c+n-k-1)}{(b+n-k-1)} \frac{(k+1) a_{k+1}}{k a_{k}} b_{k}, & \forall 1 \leq k \leq n-2 ; \\
b_{n}=\frac{c}{1+b-c} \frac{n a_{n}}{(n-1) a_{n-1}} b_{n-1}, & k=n-1 .\end{cases}
\end{gathered}
$$

Now,

$$
\begin{aligned}
& \frac{1}{(2+\alpha)^{\lambda}(2+\beta)^{\mu}} b_{1}-b_{2} \geq \frac{1}{2^{\lambda+\mu+2}}(2-\alpha \lambda)(2-\beta \mu) \\
& \cdot b_{1}-\left(\frac{c+n-2}{b+n-2}\right) \frac{2 a_{2}}{a_{1}} b_{1} \\
& =\frac{b_{1}}{2^{\lambda+\mu+2}}[(2-\alpha \lambda)(2-\beta \mu) \\
& \left.-\left(\frac{c+n-2}{b+n-2}\right) \frac{2^{\lambda+\mu+3} a_{2}}{a_{1}}\right] \geq 0
\end{aligned}
$$

Further, for $2 \leq k \leq n-2$,

$$
\begin{aligned}
& \frac{(k+\alpha)^{\lambda}(k+\beta)^{\mu}}{(k+1+\alpha)^{\lambda}(k+1+\beta)^{\mu}} b_{k}-b_{k+1} \geq\left(1-\frac{\lambda}{k+\alpha}\right)(1 \\
& \left.-\frac{\mu}{k+\beta}\right) b_{k}-\frac{(c+n-k-1)}{(b+n-k-1)} \frac{(k+1) a_{k+1}}{k a_{k}} b_{k} \\
& =\frac{b_{k}}{(k+\alpha)(k+\beta)}[(k+\alpha-\lambda)(k+\beta-\mu) \\
& \left.-(k+\alpha)(k+\beta) \frac{(c+n-k-1)}{(b+n-k-1)} \frac{(k+1) a_{k+1}}{k a_{k}}\right] \\
& \geq 0
\end{aligned}
$$

For $k=n-1$,

$$
\begin{aligned}
(1+ & \left.\frac{1}{n-1+\alpha}\right)^{-\lambda}\left(1+\frac{1}{n-1+\beta}\right)^{-\mu} b_{n-1}-b_{n} \\
\geq & \left(1-\frac{\lambda}{n-1+\alpha}\right)\left(1-\frac{\mu}{n-1+\beta}\right) b_{n-1} \\
& -\frac{c}{(1+b-c)} \frac{n a_{n}}{(n-1) a_{n-1}} b_{n-1},
\end{aligned}
$$

which is nonnegative. Following the same argument as in Theorem $5, z s_{n}^{(b-1, c)}(f, z)$ is typically real which completes the proof.

Remark 23. Note that we have no result for the close-toconvexity of $s_{n}^{(b-1, c)}(f, z)$ with respect to the starlike functions $z /(1-z)^{2}$ and $z /\left(1-z+z^{2}\right)$. Although there are not many results in the literature for close-to-convexity with respect to $z /\left(1-z+z^{2}\right)$, it will be interesting if one can find the results in this direction.

\section{Conflicts of Interest}

The authors declare that they have no conflicts of interest.

\section{Acknowledgments}

The first author acknowledges the Council of Scientific and Industrial Research, India (Grant code 09/143(0827)/2013EMR-1), for financial support to carry out the above research work.

\section{References}

[1] A. Gluchoff and F. Hartmann, "Univalent polynomials and nonnegative trigonometric sums," The American Mathematical Monthly, vol. 105, no. 6, pp. 508-522, 1998.

[2] S. Ruscheweyh and L. Salinas, "Stable functions and Vietoris' theorem," Journal of Mathematical Analysis and Applications, vol. 291, no. 2, pp. 596-604, 2004.

[3] B. Dumitrescu, Positive Trigonometric Polynomials and Signal Processing Applications, Signals and Communication Technology, Springer, Dordrecht, Germany, 2007.

[4] D. K. Dimitrov and C. A. Merlo, "Nonnegative trigonometric polynomials," Constructive Approximation, vol. 18, no. 1, pp. 117143, 2002.

[5] J. J. Fernández-Durán, "Circular distributions based on nonnegative trigonometric sums," Biometrics, vol. 60, no. 2, pp. 499503,2004 .

[6] G. Gasper, "Nonnegative sums of cosine, ultraspherical and Jacobi polynomials," Journal of Mathematical Analysis and Applications, vol. 26, pp. 60-68, 1969.

[7] R. Askey and G. Gasper, "Inequalities for polynomials," in The Bieberbach conjecture, A. Baernstein II, D. Drasin, P. Duren, and A. Marden, Eds., Math. Surveys and Monographs, no. 21, pp. 732, American Mathematical Society, Providence, RI, USA, 1986.

[8] D. K. Dimitrov, "Extremal positive trigonometric polynomials," in Approximation Theory, pp. 136-157, DARBA, Sofia, 2002.

[9] Sz. Gy. Révész, Extremal problems for positive definite functions and polynomials (PHD Thesis), Thesis for the degree Doctor of Academy, Budapest, Hungary, 2009.

[10] L. Vietoris, "Über das Vorzeichen gewisser trigono metrischer Summen," Sitzungsberichten der Österreichischen Akademie der Wissenschaften, pp. 125-135, Springer, Berlin, Germany, 1958.

[11] S. Koumandos, "An extension of Vietoris's inequalities," The Ramanujan Journal, vol. 14, no. 1, pp. 1-38, 2007.

[12] G. Brown, F. Dai, and K. Wang, "Extensions of Vietoris's inequalities I," The Ramanujan Journal, vol. 14, no. 3, pp. 471507, 2007.

[13] P. Sangal and A. Swaminathan, "Extension of Vietoris' inequalities for positivity of trigonometric polynomials," http://arxiv .org/abs/1705.03759.

[14] P. L. Duren, Univalent Functions, Springer, Berlin, Germany, 1983.

[15] A. W. Goodman, Univalent Functions, vol. 1, Mariner Publishing, Tampa, FL, USA, 1983.

[16] C. Pommerenke, Univalent Functions, Vandenhoeck \& Ruprecht, Göttingen, Germany, 1975.

[17] S. Ruscheweyh, "Linear operators between classes of prestarlike functions," Commentarii Mathematici Helvetici, vol. 52, no. 4, pp. 497-509, 1977. 
[18] S. Ruscheweyh, Convolutions in Geometric Function Theory, Séminaire de Mathématiques Supérieures, Les Presses de I’Universite. de Montreal, Montreal, QC, Canada, 1982.

[19] S. Ruscheweyh, "Coefficient conditions for starlike functions," Glasgow Mathematical Journal, vol. 29, no. 1, pp. 141-142, 1987.

[20] S. R. Mondal and A. Swaminathan, "On the positivity of certain trigonometric sums and their applications," Computers \& Mathematics with Applications, vol. 62, no. 10, pp. 3871-3883, 2011.

[21] Z. Nehari, Conformal Mapping, McGraw-Hill, New York, NY, USA, 1st edition, 1952.

[22] P. Sangal and A. Swaminathan, "Starlikeness of gaussian hypergeometric functions using positivity techniques," Bulletin of the Malaysian Mathematical Sciences Society, pp. 1-15, 2016.

[23] S. Ruscheweyh, "Geometric properties of the Cesàro means," Results in Mathematics, vol. 22, no. 3-4, pp. 739-748, 1992.

[24] M. R. Agrawal, P. G. Howlett, S. K. Lucas, S. Naik, and S. Ponnusamy, "Boundedness of generalized Cesáro averaging operators on certain function spaces," Journal of Computational and Applied Mathematics, vol. 180, no. 2, pp. 333-344, 2005.

[25] K. Stempak, "Cesàro averaging operators," Proceedings of the Royal Society of Edinburgh, Section: A Mathematics, vol. 124, no. 1, pp. 121-126, 1994.

[26] R. M. Ali, S. R. Mondal, and V. Ravichandran, "Zero-free approximants to derivatives of prestarlike functions," Journal of Inequalities and Applications, vol. 2013, article 401, 2013.

[27] J. Bustoz, "Jacobi polynomial sums and univalent Cesàro means," Proceedings of the American Mathematical Society, vol. 50, pp. 259-264, 1975.

[28] J. L. Lewis, "Applications of a convolution theorem to Jacobi polynomials," SIAM Journal on Mathematical Analysis, vol. 10, no. 6, pp. 1110-1120, 1979. 


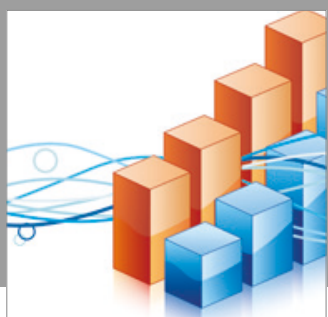

Advances in

Operations Research

vatersals

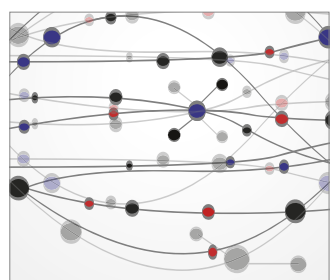

\section{The Scientific} World Journal
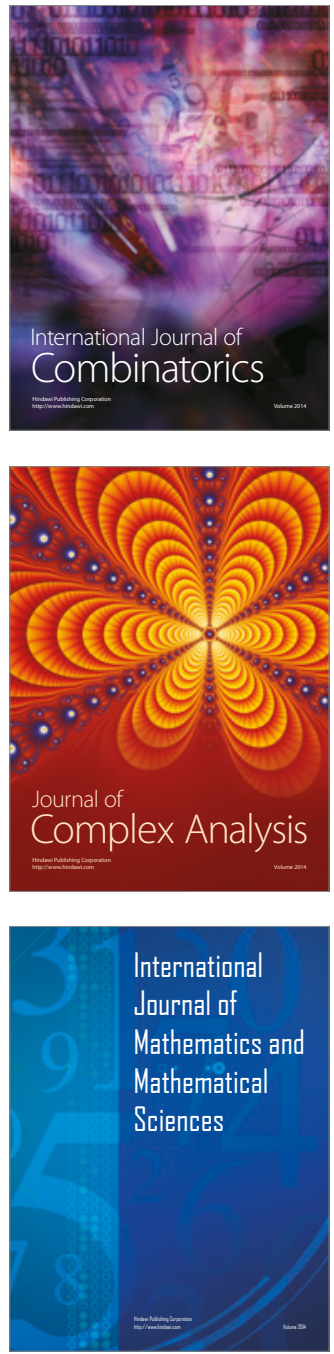


Algebra

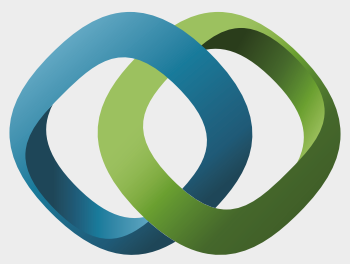

\section{Hindawi}

Submit your manuscripts at

https://www.hindawi.com
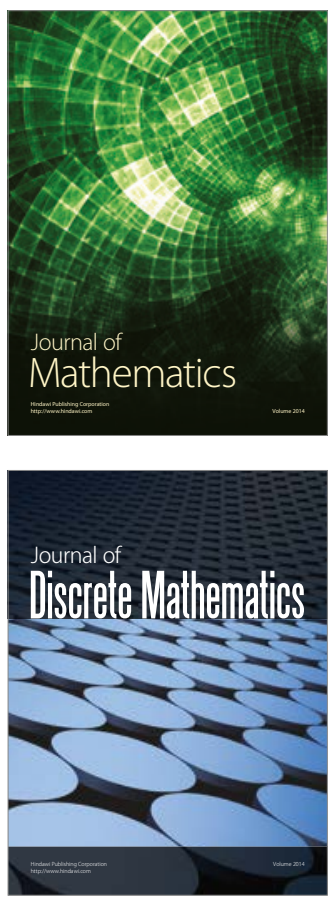

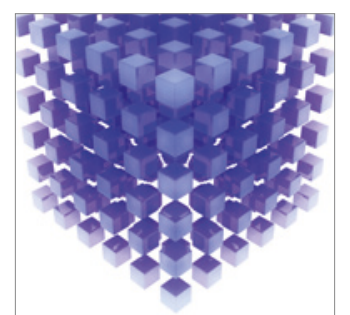

Mathematical Problems in Engineering
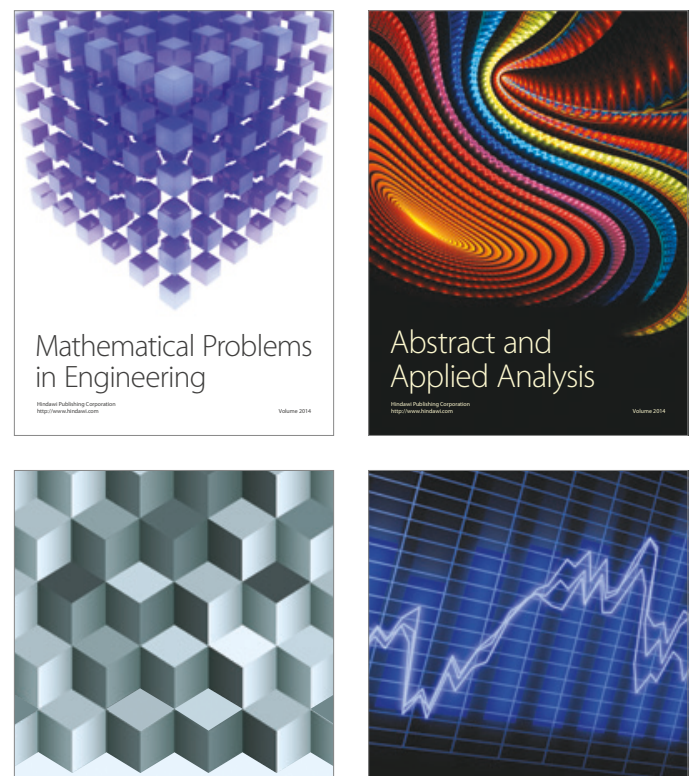

Journal of

Function Spaces

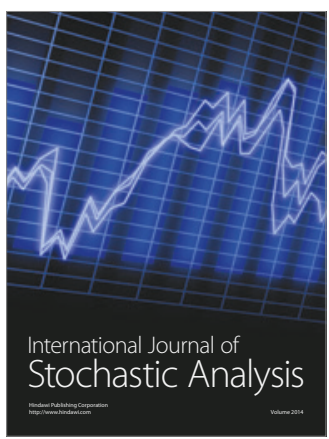

Probability and Statistics
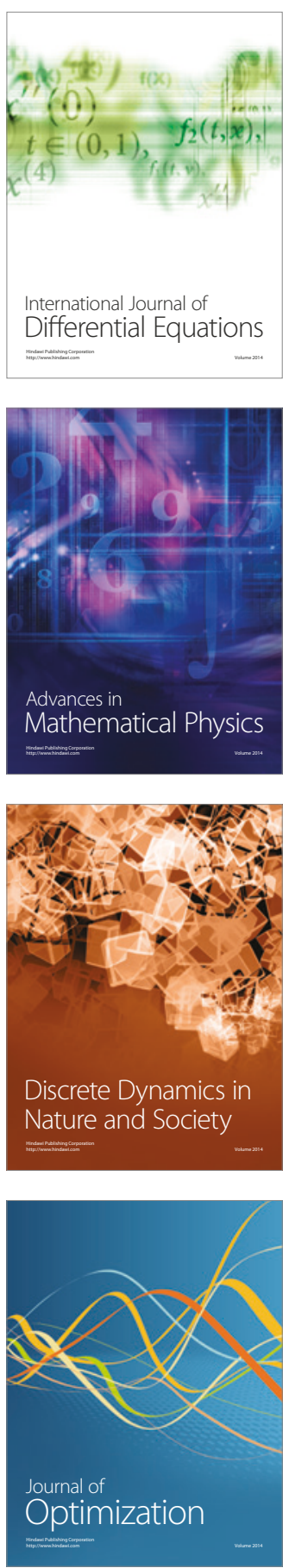